\title{
Waste Management in loT- Facilitated Smart Cities- A Suvery
}

\author{
Swati K. Rajput \\ Assistant Professor \\ DYPSOEA, Ambi, Talegaon
}

\author{
Madhavi Patil \\ Assistant Professor \\ DYPSOEA, Ambi, Talegaon
}

\begin{abstract}
The new period of Web and Internet of Things (IoT) paradigm is being enabled by the proliferation of various devices like RFIDs, sensors, and actuators. Smart devices (devices having significant computational abilities, converting them to 'smart things') are embedded in the environment to monitor and collect ambient information. In a city, this leads to Smart City backgrounds. Intelligent services could be offered on top of such information related to any aspect of humans' activities. A typical example of services offered in the framework of Smart Cities is IoT-enabled waste management. Waste management involves not only the collection of the waste in the field but also the transport and disposal to the appropriate locations. In this paper, we present a broad and thorough survey of ICT-enabled waste management models. Specifically, we focus on the implementation of smart devices as a key enabling technology in existing waste management. We report on the strengths and weaknesses of various models to reveal their characteristics. This review sets up the basis for delivering new models in the domain as it reveals the needs for defining novel outline for waste management.
\end{abstract}

\section{General Terms}

Waste Identity, Weight, and Stolen Bins Identification System (WIWSBIS), K- nearest neighbor (KNN), Receiver Operating Characteristic (ROC)

\section{Keywords}

Radio Frequency Identification (RFID), Waste Identity, Weight, and Stolen Bins Identification System (WIWSBIS), Ubiquitous Sensor Network (USN), Gray Level Aura Matrix (GLAM), Receiver Operating Characteristic (ROC).

\section{INTRODUCTION}

BY 2050, the huge amount of earth population (i.e., 70\%) will move to urban areas, thus, forming vast cities. Such cities require a smart defensible infrastructure to manage citizense needs and offer vital and more forward-thinking services. The acceptance of Future Internet technologies enhanced by the use of the Internet Protocol (IP) on several wireless sensors allows the Internet of Things (IoT) paradigm. Several sensors have the opportunity to be part of Wireless Sensor Networks (WSNs). When WSNs are applied in a city, they are responsible for collecting and processing ambient information and, thus, to upgrade legacy city infrastructure to the so-called Smart Cities (SCs). A definition of the concept of SC is provided in: "A Smart City is a city well performing in a forward-looking way in the following fundamental components (i.e., Smart Economy, Smart Mobility, Smart Environment, Smart People, Smart Living, and Smart Governance), built on the "smart" arrangement of endowments and actions of self-decisive, independent and aware citizens".
This explanation incorporates the fundamental component of a smart environment which is mainly adopted for systems dealing with environmental pollution. The concept of smart environments depicts the ambient intelligence found in a SC through the adoption of smart devices and wireless networks. This way, intelligent applications could be delivered on top of such infrastructures. WSNs are capable of reforming activities in a SC in every aspect of daily life. In this paper, we focus on a specific application domain, waste management. The efficient management of waste has a significant impact on the class of life of citizens. The purpose is that waste disposal has a clear linking with destructive impacts in the environment and thus on citizens ${ }^{\text {ee }}$ health. In this paper, the advantage of study on waste management problem in the city of St. Petersburg, Russia. St. Petersburg is a city of 5 million citizens cover a total area of 1,439 square kilometers, a density of 3,391 citizens per square kilometer. On average, solid waste produced in the city is 1.7 million tons per year. The daily amount of municipal solid waste generated is 0.93 kilograms per citizen. On a daily basis, the municipality of St. Petersburg uses 476 waste assembly trucks with a capacity of 5 tons per truck. The fuel consumed in one year is, on average, 1.8 million liters. The typical costs spent for fuel in one year for waste collection is more than 1 million US dollars. Finally, the traffic congestion caused by the fleet of waste collection trucks at rush hours is significant due to the narrow roads and small backyards, causing indirect problems in citizens"e activities. Obviously, it is serious to efficiently manage the waste disposed in every location of a SC not only focusing on the collection activities but also on its transport and recycling.

This paper models the waste management as a set of services on top of an IoT infrastructure in a SC. These services cover the following parts of a waste management scheme: (i) waste collection arrangement and employment (e.g., routing solutions for collection trucks, dynamic adaptation of routes); (ii) transport of waste to specific localities (e.g., routing according to the type of waste); (iii) recycling and preparation for re-use. In this paper, the focus on the first type of services i.e., efficient organization of waste collection activities. In this paper also focus on dynamic models on contemporary waste collection with the propagation of Radio Frequency Identification (RFID), sensors and actuators. Several devices have been adopted for enabling the efficient implementation of the dynamic waste collection e.g., RFID tags, sensors and actuators. With the term dynamic, we denote the ability of a system to change, in real time, the factors and the plans that affect the collection of waste during the collection action. Such functionalities could be incorporated into an intelligent transportation framework that results in real time directions provided to the collection trucks. Intelligent transportation contributes to dynamic waste collection since it uses smart vehicle infrastructure, which is incorporated in this survey. 
Specifically, in this paper a comprehensive survey on the adoption of Information and Communication Technologies (ICT) in waste management models focusing on the modern ICT tools and technologies. Here, survey a substantial body of knowledge, thirty-two case studies. Among them, only six models involve an IoT-enabled technology. The argument that waste management solutions should be adopted as the backend middleware to support further inference and reasoning on top of the data coming from sensors. In this paper also a discussion on a taxonomy of the studied models and, thus, to compare the strengths and weaknesses of each one. The rest of the paper is structured as follows Section 2 reports on the survey of relevant models and the relative valuation. Section 3 concludes the paper and deliberates future work.

\section{SURVEY AND COMPARATIVE STATEMENT}

The efforts reviewed are compared according to the above proposed taxonomy while their strengths and weaknesses are clearly stated. These research efforts cover more than ten years of research in the area of ICT waste management. Here survey thirty- two papers while only six exploit the IoT Technology as the back- end system for delivering intelligent applications. The distribution of the papers by the publication year is depicted. Taking advantage of this survey, we propose a reference model which exploits IoT capabilities combining the strengths and eliminating the weaknesses of the surveyed models.

\section{A. Models that assume Capacity, Weight, Temperature, Humidity and Chemical Sensors ic}

Researchers adopt capacity, weight, temperature, humidity and chemical sensors for solid waste collection. Specifically, the authors propose a municipal solid waste platform exploiting recycling collection information based on IoT Technology. The paper presents a model for waste collection, transportation, recycling and processing. A management information platform based on IoT Technology is proposed which serves a waste collection model in the city of Wuhan. The outcomes of the research help municipal authorities to use efficiently the information produced in every stage of the waste collection process and, finally, achieve the goal of an intelligent cycle. The authors propose a dynamic optimization model for solid waste recycling. The paper presents a model for recycling materials and dynamic optimization. A dynamic decision model characterized by state variables is developed; it corresponds to the quality of waste in each bin on a daily basis. The model controls the variables, thus determining the quantity of the collected materials. It is also responsible for calculating the routes for each collection truck. An objective function is defined that is studied to minimize the sum of the collection costs. The decision model is integrated in a DSS that is enabled with a GIS. A case study in the municipality of Cogoleto, Italy is presented which proves the effectiveness of the proposed model. Specifically, the net benefits of the optimized waste collection are 2.5 times greater than the estimated current policy.

\section{B. Models that assume Capacity, Weight, Temperature, Humidity and Pressure Sensors}

The researchers focus on the use of capacity, weight, temperature, humidity and pressure sensors for solid waste collection. More specifically, the authors have proposed a sensorized waste collection container for content guesstimate and collection optimization. The paper presents the design and implementation of a suitable urban solid waste system which can predict the quantity and multiplicity of solid waste. They adopt measures to correlate the capacity of solid waste with residential population and consumer index at different seasons of the year. The system incorporates an intelligent and sensorized bin in order to exploit data used for further statistical inference processes. The proposed bin is prepared and tested in the Pudong New Area, Shanghai. The authors have proposed a solid waste collection architecture using WSNs. The paper presents a WSN model as a key enabling technology for smart implementation to face the waste collection problem in an urban area. Architecture is proposed in order to improve and optimize the on-site handling and transportation during the waste collection process. The system architecture incorporates sensor nodes and uses data transfer nodes in order to provide data measurements, collected from bins, to a remote server. A remote monitoring web application is also incorporated, providing user the capability of interacting with the system through a Web browser. A DSS is also available aiming to provide solutions to resources organization difficulties.

\section{Models that assume Capacity and Weight Sensors}

The next set of papers in the area consists of five research efforts where the authors also deal with capacity and weight sensors. More specifically, in the authors propose a collectionmonitoring model for early detection and evaluation of waste through sensorized bins. The research extends by describing a innovative application used to monitor the municipal solid waste, based on distributed sensor technology and GIS. The paper presents a model tested and evaluated in Pudong, Shanghai (PR China). The waste collection system has specific monitoring requirements related to the rapidly increasing rate of waste production. The paper focuses on the quantitative measurement of the waste present at each bin and identification of different types of waste collected in the bins. The model incorporates a network of sensorized bins linked to a DSS. Bins are equipped with a set of sensors which provide real data to the system while trucks incorporate GPS for real time monitoring. The collected data are integrated into an existing application used by municipality authorities. Evaluation is done on real data flow (the software analytics part) from the network of bins as well as in terms of optimization functions such as dynamic scheduling and routing. Research uses obtained outcomes related with waste weight and volume as parameters for optimizing the proposed dynamic routing model and evaluating the material density in the bins. The authors have proposed an energy consumption model for augmenting solid waste collection, which is applied in a large city area. The research extends by providing three models for dynamic scheduling and routing improved with path optimization. The paper presents an improved waste collection model which is enabled with capacity and weight data collected for bins. In addition, it is exploited energy utilization related to the trucks, which optimize municipal solid waste processes. A genetic algorithm and a simulation model are adopted to optimize the collection route. There are incorporated in both a DSS and a GIS for efficient administrative management of the system. The proposed model is verified through a case study in Pudong. The results are very promising for waste collection and transport by achieving energy utilization. The authors have projected a model for analyzing the impact of solid waste Source Segregation (SS) intensity on fuel consumption along with the collection costs. The paper presents an evaluation on the fuel consumed and an analysis on the collection costs of solid waste collection. A recreation model is incorporated as well. The proposed model can calculate the time spent, the waste capacity collected and the fuel consumed for a specific waste 
collection route. The initial value of SS intensity is set to $25 \%$ where the assessed model error was less than 1.2. The simulation scenarios are held with different values of $25 \%$, $30 \%, 35 \%$, and $52 \%$ of SS intensity. An increase in the average fuel consumed by collection trucks is observed started form 3.3 lit/ton for $25 \%$ SS intensity and reached up to 3.8 lit/ton for SS intensity of $52 \%$. In addition, direct collection costs; with crews and truck purchase, ranged from $40 € /$ ton to $70 € /$ ton, respectively, for $25 \%$ and $52 \%$ of the SS intensity. The research proves that the increased values of fuel expended and collection costs depend on the density of the waste collected, on the group truck compaction ratio and on the Waste Collection Truck Utilization Factor (WCTUF).

\section{Models that approve Capacity and Pressure Sensors}

Researchers adopt capacity and pressure sensors for solid waste collection. The authors in propose an economic performance comparison between pneumatic and door-to-door waste collection systems in existing urban areas. The paper presents an analysis on how a hypothetical stationary pneumatic waste collection system can be compared economically to a old-style truck-operated door-to-door collection system. In heavily populated urban areas both pneumatic and door-to-door waste collection systems face disadvantages. Specifically, buildings and fixed city infrastructure increase the installation costs of a pneumatic system in existing residential areas. Inversely, the limited space for waste transportation trucks and bins cause problems for truck-operated waste collection systems.

The paper uses a method for analyzing the cost effects of the waste collection systems, which takes into consideration the monetized environmental effects of both waste collection systems. Door-to- door waste collection is proved to be economically six times more superior; approximately. In the performed analysis the dominant cost factor is the large investment cost of the pneumatic system. Another significant impact factor is the economic value of land, because it is able to reverse the results of the analysis; in case that the value of land saved with pneumatic system is sufficiently high. The authors propose an automatic waste collection system based on Ubiquitous Sensor Network (USN). The paper presents a novel approach to collecting municipal solid waste in residential and commercial buildings using IoT Technology. USN technology eliminates web of wires while increases connectivity of devices, thus, providing an easy extension of the network. An USN architecture is proposed which is experimentally evaluated with real time scenarios in the city of Seoul, South Korea. The system is composed of integrated WSNs, gateways and specially designed software. The research covers all the processes of waste collection including waste loading and transportation in pneumatic pipes from depots to dumps. The proposed system is compared with a conventional wired network and proved to perform in-time fast waste collection. Furthermore, it has low installation, operational and maintenance costs; while it is scalable and reliable. Compared with our taxonomy, organic, glass, paper, plastic and metal waste bins are used which are located outdoors in both models Pneumatic pipes infrastructure is incorporated and the fleet of trucks is homogenous. Multiple dumps are used. Recycling of inorganic waste and further processing of organic waste are supported. WSNs are implied in both efforts while dynamic scheduling and routing are defined with social context being supported. Finally, models were evaluated on real experimental data retrieved in the cities of Helsinki and Seoul.
The strengths of the models are the detailed analysis of the hypothetical stationary pneumatic waste collection system, the comparison with a traditional truck-operated door-to-door collection system by means of economics efficiency, the welldefined USN architecture and the exploitation of IoT Technology.

\section{E. Models that assume RFIDs}

In the researchers incorporate extensively RFIDs for tagging and identification as part of the solid waste collection infrastructure. Specifically, the authors have proposed an RFID-based real time smart waste collection system. The paper presents the use of RFID and weight sensor technology as part of waste collection. The IoT architecture is proved to decrease waste collection operational costs and enables automating and streamlining waste identification for recycling and further processing of waste. Furthermore weight measurement processes are incorporated in the proposed real time waste collection system. The paper concludes with an application that exploits RFID and weight sensor data in order to define an automatic Waste Identity, Weight, and Stolen Bins Identification System (WIWSBIS).

The authors have proposed a waste collection multi-objective model based on real time traceability data. The paper presents the investment, operational and environmental costs related with the waste collection. A variety of IoT mechanisms is used in order to provide the system with real time data used to implement an efficient and innovative waste collection routing model. The research states that knowing the real time data of each truck and the real replenishment level at each bin enables dynamic scheduling and routing in relation with a waste generation pattern. Dynamic models can be optimized incorporating further information about total distance covered, necessary number of trucks and the environmental impact. It is also described a framework about enabling traceability and monitoring in the optimization of solid waste collection. The framework is integrated with an innovative dynamic routing model which uses real time traceability data from an Italian city of 100,000 inhabitants. The model was tested and validated using simulation; while it is also performed an economic feasibility study. The authors have proposed an automated bin level detection system using a gray level aura matrix.

The research extends by introducing an advanced image processing approach integrated with communication technologies and a camera for bin level detection. The paper presents the environmental concerns associated with bins and the kind of waste disposed in them. A Gray Level Aura Matrix (GLAM) approach is used in order to extract the bin image texture. GLAM neighboring system parameters are tuned to determine their optimal values. The system is evaluated by training and testing the extracted image with Multi-Layer Perceptions (MLPs) and K- nearest neighbor $(\mathrm{KNN})$ classifiers. The paper proves that the proposed system performance is robust and can be applied to different kinds of waste and bin level detection under various conditions. The authors have proposed a novel dynamic scheduling and routing model incorporating GIS, which reduce the operation costs and pollutant emissions of MSW collection. The paper presents an innovative methodology for the reduction of the operational costs and pollutant emissions involved in the waste collection and transportation. Dynamic scheduling and routing are combined for efficient waste collection. Dynamic routing is based on historical data initiated by the filling rate of each bin individually in order to establish the daily routes of collection points to be visited. This approach is more 
realistic compared with the usual assumption of a single average fill-up rate because it is common to all system bins. Dynamic routing enables forward planning of the dynamic scheduling; which permits a better system management. The optimization process of the negotiated routes incorporates a GIS and uses total spend time and covered distance as optimization principles. The model also reflects variables related to fuel consumption, impurity productions, truck speed and capacity transported as part of the optimization process. Experimental evaluation is performed for the case of glass waste collection and transportation in the city of Barreiro in Portugal. The research isolates the influence of the dynamic load; on fuel consumed and pollution emitted, by incorporating a sensitivity analysis of the truck loading process. Specifically, it was tested two scenarios of waste collection: (i) one with the collected waste capacity increasing exponentially along the collection path, and (ii) the other assuming that the collected waste capacity decreasing exponentially along the same path. Optimization performed on operation costs of labor, truck maintenance and fuel consumptions; as well as pollutant emissions, are proved to have beneficial impacts regardless of the optimization criterion used. Nonetheless, this impact is particularly relevant when optimizing for time; yielding substantial improvements to the existing system. In addition, it is proved that dynamic loading process of the collection trucks impacts on both fuel consumed and pollution emitted. The authors have proposed a solid waste dumping system using mobile ad-hoc networks. The paper presents a model for waste collection of bins, distributed in a highly densely populated city in India. A dynamic multi-hop network that can provide real time information to municipal authorities is formed. The system is able to monitor online and visualize the status of the bins for further use; due to a sensors and adhoc transceivers embedded in the bins. The authors have proposed a model for assessing and improving management practices in planning packaging waste collection systems. The paper presents a packaging model for waste collection systems that incorporates depots as transfer and sorting stations. During the application of the current strategy each depot is managed independently and not as a part of the whole system. The research proposes four tactical and operational practices which contribute to the independent management of each depot. The model applies these follows to the proposed infrastructure and evaluates their impacts on the total collection cost. A design methodology is developed to plan service areas, dynamic scheduling and routing. The model exploits alternative solutions in managing the system as a whole. The methodology is applied to real use cases of a waste collection and packaging company which serves 7 municipalities in mainland Portugal. The results were encouraging because of the decreased total cost of the system. The decrease in total cost was achieved because of the significant savings obtained in terms of minimizing: (i) the total distance covered, and (ii) the number of trucks required for waste collection.

\section{F. Models that agree WSNs}

The next set of papers, i.e., also focuses on the extensive use of WSNs. More specifically, the authors propose a solid waste collection system, which incorporates an effective dynamic scheduling, and routing model. it is presented the Malmoe simulation model suggested in the paper. The paper presents capacity sensors and wireless communication equipment embedded to recycling bins. In the IoT infrastructure, the research effort provides waste collection operators and access to real time information about of the status of each bin. The architecture uses analytical modeling and discrete-event simulation to evaluate dynamic scheduling and routing by utilizing real- data. Furthermore, the discussed research performs an empirical simulation based on real experimental data from a recycling station in Malmoe, Sweden. During this study it is proved that dynamic scheduling and routing models have lower operational costs, shorter collection and hauling distances, and reduced labor hours compared with static models. the authors have proposed an eco-design Machine-toMachine (M2M) system that contributes to waste glass collection i.e., the Product Service System (PSS). The research methodology is based on the Life Cycle Analysis (LCA) model. The provided results help to understand the mechanisms of the PSS while research explains how the proposed infrastructure impacts the environment. The paper examines the impact of system parameters to eco-design and dimensioning of the PSS. The study incorporates mutualisation analytics to reduce environmental impacts while limiting data exchanges. Additionally, the authors propose a wireless platform, called WEKO, for monitoring the recycling spots. The paper presents an embedded WSN for monitoring the level of bins located in recycling spots. The system interacts with a remote central station when a bin reaches some filling threshold, thus, avoiding checking the spot if the bin is full while ensuring that the recycling spot is kept clean. The wireless nature of the system imposes energy supply requirements which are covered with specific batteries with a life time of at least six years. Data collected from sensors are captured by a monitoring platform located in the remote central station for further processing. The authors propose an inventory routing model for dynamic waste collection. The paper presents the process of waste collection incorporating underground bins equipped with sensors. The research treats the waste collection routing as a reverse inventory dynamic routing problem taking into deliberation decisions involving routing and bin selection. The model is able to cope with uncertainty on the bins capacity and fluctuations of routes; due to daily and periodic effects. The need of an anticipatory policy which will balance the workload over time is also stated. The paper proposes a computational efficient heuristic which consists of several tunable parameters depending on a weekly schedule. The parameters are tuned using optimal learning techniques as part of a simulation model.

\section{G. Models that agree Actuators}

The researchers incorporate actuators as part of the solid waste collection infrastructure. Specifically, the authors propose a bin level detection model based on a gray level cooccurrence matrix feature (GLCM) extraction approach. The research extends by focusing on solid waste bin level detection and classification using the GLCM feature extraction. The paper also presents a set of GLCM displacement and quantization parameters along with the number of textural features which are tuned to determine the best parameter values of the bin images. Parameters and textural features are incorporated in the GLCM database adopted for reasoning. Image classification and grading is based on training and testing of MLPs and KNN classifiers. The proposed model can be used in bin level classification and grading, thus, providing a robust solution for bin detection, monitoring and management. In the authors propose a solid waste bin detection and classification model using Dynamic Time Warping (DTW) and MLP classifiers. The research extends by focusing on image processing solutions for solid waste collection. The paper presents the process of capturing bin image with a camera embedded in the bin. Specifically, during capturing the bin image the position of the camera should be focus on getting a centralized image 
of the bin area. The captured image should be further processed in order to correctly estimate the waste capacity in the bin. The research uses DTW for detecting and cropping the bin area; while Gabor Wavelet $(\mathrm{GW})$ is combined for feature extraction of the bin image. The features extracted are then used to train a MLP classifier which is adopted to classify the bin level and perform guesstimates about the capacity of the waste in the bin. The classifier performance is evaluated with Receiver Operating Characteristic (ROC) curves and proved to be efficient with respect to the accuracy (i.e., level estimation of 98.5\%). The authors propose an efficient waste collection model incorporating shortest path semi-static and dynamic routing.

\section{SORTING AND PROPORTIONAL ASSESSMENT}

The summary of the comparative assessment performed on the survey is presented. Total Fifteen research efforts, are examined and their strengths and weaknesses are revealed. Through the provided survey, we try to classify each model according to our taxonomy developed to depict important parts of the proposed systems. Concerning the physical infrastructure, organic bins are used in 22 models, glass bins in 20 models, paper bins in 25 models, plastic bins in 23 models, metal bins in 21 models and toxic bins in 4 models. Waste bins are located outdoors in 28 models while they are underground in 2 models. In just one model, bins are located both outdoors and underground. Pneumatic pipes are incorporated in 2 models; the fleet of trucks is homogenous in 22 models while it is heterogeneous in 9 models. Depots are incorporated in 6 models while they are absent in 25 models. Dumps are single in 18 models while they are multiple in 13 models. Recycling of inorganic waste is supported in 17 models and further processing of organic waste is supported in 8 models. The IoT Technology includes RFIDs (14 models), capacity sensors (30 models), weight sensors (12 models), temperature sensors (5 models), humidity sensors (6 models), chemical sensors (5 models) and pressure sensors (5 models). WSNs are adopted in 14 models and actuators are incorporated in 7 models. Cameras are incorporated in 6 models and GPS is incorporated in 16 models. In the category of software analytics, a DSS is incorporated in 9 models, GIS is discussed in 15 models, dynamic scheduling is defined in 16 models and dynamic routing is defined in 25 models. The social context is supported in 6 models. Synthetic experimental data are adopted for 7 models while real experimental data are adopted for the evaluation of 19 models. Moreover, 5 models are evaluated on both synthetic and real experimental data.

\section{CONCLUSIONS}

This survey's focus is on more energy-efficient IoT as an enabler of various applications including waste management. Specifically, it aims to present a large set of models dealing with the efficient waste management. Special attention is paid on the waste collection. In this paper, efforts are presented for the intelligent transportation within the context of IoT and Smart Cities for waste collection. Here the proposal of an inductive taxonomy to perform comparative assessment of the surveyed models. The focus only on efforts that incorporate ICT models for waste collection in SC. We deliver the strengths and weaknesses of the surveyed models. Finally, our future work is focused on the definition of an effective IoTenabled model for waste collection, which will touch on the incorporation of high capacity waste trucks as mobile depots. In addition, waste bins are placed to optimize coziness of residents. However, as part of the future work we will be looking at bin connectivity restrictions that may affect their location, for example, the output power of a communicating sensor would need to be set too high which may drain the battery quicker. In this case, the bin may be placed somewhere where energy consumption is more efficient.

\section{REFERENCES}

[1] M. Fazio, M. Paone, A. Puliafito, and M. Villari. "Heterogeneous Sensors Become Homogenous Things in Smart Cities", IEEE 6th International Conference on Innovative Mobile and Internet Services in Ubiquitous Computing (IMIS), 2012, pp. 775-780.

[2] C. Balakrishna, "Enabling Technologies for Smart City Services and Applications", IEEE 6th International Conference on Next Generation Mobile Applications, Services and Technologies (NGMAST), 2012, pp. 223227.

[3] S. Suakanto, S. H. Supangkat, Suhardi, and R. Sarasgih, "Smart City Dashboard for Integrating Various Data of Sensor Networks", IEEE International Conference on ICT for Smart Society (ICISS), 2013, pp. 1-5.

[4] R. Carli, M. Dotoli, R. Pelegrino, and L. Ranieri, "Measuring and Managing the Smartness of Cities: A Framework for Classifying Performance Indicators", IEEE International Conference on Systems, Man, and Cybernetics (SMC), 2013, pp. 1288-1293.

[5] C. Tao, and L. Xiang, "Municipal Solid Waste Recycle Management Information Platform Based on Internet of Things Technology", IEEE International Conference on Multimedia Information Networking and Security (MINES), 2010, pp. 729-732.

[6] D. Anghinolfi, M. Paolucci, M. Robba, and A. C. Taramasso, "A dynamic optimization model for solid waste recycling", Waste Management, vol. 33 (2), 2013, pp. 287-296.

[7] M. A. Hannan, M. Arebey, R. A. Begum, and H. Basri, "Radio Frequency Identification (RFID) and communication technologies for solid waste bin truck monitoring system", Waste Management, vol. 31 (12), 2011, pp. 2406-2413.

[8] K. Lingaraj, R. V. Biradar, and V. C. Patil. A survey on middleware challenges and approaches for wireless sensor networks. In 2015 International Conference on Computational Intelligence and Communication Networks (CICN), pages 56-60, Dec 2015. Institute of Electrical and Electronics Engineers Inc. https://doi.org/10.1109/CICN.2015.20

[9] F. Vicentini, A. Giusti, A. Rovetta, X. Fan, Q. He, M. Zhu, and B.Liu, "Sensorized waste collection container for content

estimation and collection optimization", Waste Management, vol. 29 (5), 2009, pp. 1467-1472.

[10] S. Longhi, D. Marzioni, E. Alidori, G. D. Buo, M. Prist, M. Grisostomi, and M. Pirro, "Solid Waste Management Architecture Using Wireless Sensor Network Technology", IEEE 5th International Conference on New Technologies, Mobility and Security (NTMS), 2012, pp. $1-5$.

[11] Rovetta, F. Xiumin, F. Vicentini, M. Zhu, A. Giusti, and Q. He, "Early detection and evaluation of waste through sensorized containers for a collection monitoring 
application", Waste Collection, vol. 29 (12), 2009, pp. 2939-2949.

[12] F. Xiumin, M. Zhu, X. Zhang, Q. He, and A. Rovetta, "Solid waste collection optimization considering energy utilization for large city area", IEEE International Conference Logistics Systems and Intelligent Management, 2010, pp. 1905-1909.

[13] F. D. Maria, and C. Micale, "Impact of source segregation intensity of solid waste on fuel consumption and collection costs", Waste Management, vol. 33 (11), 2013, pp. 2170-2176.

[14] K. Lingaraj, R. V. Biradar, and V. C. Patil. A survey on mobile-agent middleware design principals and itinerary planning algorithms. In 2015 International Conference on Applied and Theoretical Computing and Communication Technology (iCATccT), pages 749753, Oct 2015. Institute of Electrical and Electronics Engineers https://doi.org/10.1109/ICATCCT.2015.7456983.

[15] V. Catania, and D. Ventura, "An approach for monitoring and smart planning of urban solid waste management using smart-M3 platform", In the Proceedings of IEEE 15th Conference on Open Innovations Association FRUCT, 2014, pp. 34-31.

[16] N. Teerioja, K. Moliis, E. Kuvaja, M. Ollikainen, H. Punkkinen, and E. Merta, "Pneumatic vs. door-to-door waste collection systems in existing urban areas: a comparison of economicperformance", Waste Management, vol. 32 (10), 2012, pp. 1782-1791.

[17] W. A. Arbab, F. Ijaz, T. M. Yoon, and C. Lee, "A USN based Automatic waste collection system", IEEE 14th International Conference on Advanced Communication Technology (ICACT),2012, pp. 936-941.

[18] B. Chowdhury, and M. U. Chowdhury, "RFID-based real-time smart waste management system", IEEE Australasian Conference on Telecommunication Networks and Applications (ATNAC), 2007, pp. 175180

[19] M. Faccio, A. Persona, and G. Zanin, "Waste collection multi objective model with real time traceability data", Wast Collection, vol. 31 (12), 2011, pp. 2391-2405.

[20] M. A. Hannan, M. Arebey, R. A. Begum, and H. Basri, "An automated solid waste bin level detection system using a gray level aura matrix", Waste Management, vol. 32 (12), 2012, pp.2229-2238.
[21] Z. Zsigraiova, V. Semiao, and F. Beijoco, "Operation costs and pollutant emissions reduction by definition of new collection scheduling and optimization of MSW collection routes using GIS. The case study of Barreiro, Portugal", Waste Management, vol. 33 (4), 2013, pp. 793-806.

[22] P. Muthukumaran, and S. B. Sarkar, "Solid waste disposal and water distribution system using the mobile adhoc network" IEEE International Conference on Emerging Trends in Communication, Control, Signal Processing \& Computing Applications (C2SPCA),2013, pp. 1-4.

[23] T. R. Pereira-Ramos, M. I. Gomes, and A. P. BarbosaPovoa, "Assessing and improving management practices when planning packaging waste collection systems", Resources, Conservation and Recycling, vol. 85, 2014, pp. 116-129.

[24] O. M. Johansson, "The effect of dynamic scheduling and routing in a solid waste management system", Waste Management, vol.26 (8), 2006, pp. 875-885.

[25] K. Lingaraj et al., Eagilla: An Enhanced Mobile Agent Middleware for Wireless Sensor Networks, Alexandria Eng. $\mathrm{J}$.

(2017), http://dx.doi.org/10.1016/j.aej.2017.03.003.

[26] T. Gomes, N. Brito, J. Mendes, J. Cabral, and A. Tavares, "WECO:A wireless platform for monitoring recycling point spots", IEEE16th Mediterranean Electrotechnical Conference (MELECON),2012, pp. 468-472.

[27] M. Mes, M. Schutten, and A. Perez-Rivera, "Inventory routing for dynamic waste collection", Waste Management, vol. 34 (9), 2014, pp. 1564-1576.

[28] M. Arebey, M. A. Hannan, R. A. Begum, and H. Basri, "Solid waste bin level detection using gray level cooccurrence matrix feature extraction approach", Journal of Environmental Management, vol. 104, 2012, pp. 9-18.

[29] Lingaraj K, Sreekanth N, Kaja Moddiudin, Lokesh K M, Prashanth Keni, \& Nagaveni. (2017). Information Security Emergency Plan Management System. Advances in Intelligent Systems and Computing Springer, 508(14), 1-9. https://doi.org/10.1007/978-98110-2750-5_14.

[30] T. Anagnostopoulos, A. Zaslavsky, "Effective Waste Collection with Shortest Path Semi-Static and Dynamic Routing", IEEE $14^{\text {th }}$ International Conference on Next GenerationWired/Wireless 